\title{
On the Internal and Boundary Stabilization of Timoshenko Beams
}

Salim A. Messaoudi and Muhammad I. Mustafa

\begin{abstract}
In this paper we consider Timoshenko systems with either internal or boundary feedbacks. We establish explicit and generalized decay results, without imposing restrictive growth assumption near the origin on the damping terms.
\end{abstract}

Mathematics Subject Classification (2000). 35B37, 35L55, 74D05, 93D15, 93D20.

Keywords. Energy decay, nonlinear damping, Timoshenko.

\section{Introduction}

Timoshenko [16] gave the following system of coupled hyperbolic equations

$$
\begin{aligned}
\rho u_{t t} & =\left(K\left(u_{x}-\varphi\right)\right)_{x}, \quad \text { in }(0, L) \times(0,+\infty) \\
I_{\rho} \varphi_{t t} & =\left(E I \varphi_{x}\right)_{x}+K\left(u_{x}-\varphi\right), \quad \text { in }(0, L) \times(0,+\infty),
\end{aligned}
$$

as a simple model describing the transverse vibration of a beam. Here $t$ denotes the time variable, $x$ is the space variable along the beam of length $L$, in its equilibrium configuration, $u$ is the transverse displacement of the beam, and $\varphi$ is the rotation angle of the filament of the beam. The coefficients $\rho, I_{\rho}, E, I$ and $K$ are respectively the mass per unit length, the polar moment of inertia of a cross section, Young's modulus of elasticity, the moment of inertia of a cross section, and the shear modulus.

Kim and Renardy [5] considered (1.1) together with boundary controls of the form

$$
\begin{aligned}
K \varphi(L, t)-K \frac{\partial u}{\partial x}(L, t) & =\alpha \frac{\partial u}{\partial t}(L, t), \quad \forall t \geq 0 \\
E I \frac{\partial \varphi}{\partial x}(L, t) & =-\beta \frac{\partial \varphi}{\partial t}(L, t), \quad \forall t \geq 0
\end{aligned}
$$


and used the multiplier techniques to establish an exponential decay result for the energy of (1.1). In addition, a polynomial decay result for the energy of (1.1) was established by Yan [18] when considering the boundary conditions

$$
\begin{array}{rlrl}
K\left(\varphi(L, t)-\frac{\partial u}{\partial x}(L, t)\right) & =f_{1}\left(\frac{\partial u}{\partial t}(L, t)\right), & & \forall t \geq 0 \\
-E I \frac{\partial \varphi}{\partial x}(L, t) & =f_{2}\left(\frac{\partial \varphi}{\partial t}(L, t)\right), & \forall t \geq 0
\end{array}
$$

and $f_{1}, f_{2}$ having polynomial growth near the origin. Soufyane and Wehbe [15] established the uniform stability of (1.1), using a unique locally distributed feedback. Precisely, they considered

$$
\begin{aligned}
\rho u_{t t} & =\left(K\left(u_{x}-\varphi\right)\right)_{x}, \quad \text { in }(0, L) \times(0,+\infty) \\
I_{\rho} \varphi_{t t} & =\left(E I \varphi_{x}\right)_{x}+K\left(u_{x}-\varphi\right)-b \varphi_{t}, \quad \text { in }(0, L) \times(0,+\infty) \\
u(0, t) & =u(L, t)=\varphi(0, t)=\varphi(L, t)=0, \quad \forall t \geq 0,
\end{aligned}
$$

where $b$ is a positive and continuous function, which satisfies

$$
b(x) \geq b_{0}>0, \quad \forall x \in\left[a_{0}, a_{1}\right] \subset[0, L],
$$

and proved that the uniform stability of (1.2) holds if and only if the wave speeds are equal $\left(\frac{K}{\rho}=\frac{E I}{I_{\rho}}\right)$; otherwise only the asymptotic stability can be obtained. This result has been extended by Rivera and Racke [11] for the damping function $b=$ $b(x)$ possibly changes sign, and for a nonlinear system in [10]. Rivera and Racke [9] also treated a nonlinear system with damping effect through heat conduction of the form

$$
\begin{aligned}
& \rho_{1} u_{t t}-\sigma\left(u_{x}, \varphi_{x}\right)_{x}=0, \quad \text { in }(0, L) \times(0,+\infty) \\
& \rho_{2} \varphi_{t t}-b \varphi_{x x}+K\left(u_{x}+\varphi\right)+\gamma \theta_{x}=0, \quad \text { in }(0, L) \times(0,+\infty) \text {, } \\
& \rho_{3} \theta_{t}-K \theta_{x x}+\gamma \varphi_{x t}=0, \quad \text { in }(0, L) \times(0,+\infty),
\end{aligned}
$$

where $\theta$ is the difference temperature. Under appropriate conditions on the nonlinearity, they proved an exponential decay result for the case of equal wave speeds $\left(\frac{K}{\rho_{1}}=\frac{b}{\rho_{2}}\right)$. Raposo et al. [12] considered the following system

$$
\begin{aligned}
\rho_{1} u_{t t}-K\left(u_{x}-\varphi\right)_{x}+u_{t}=0, & \text { in }(0, L) \times(0,+\infty) \\
\rho_{2} \varphi_{t t}-b \varphi_{x x}-K\left(u_{x}-\varphi\right)+\varphi_{t}=0, & \text { in }(0, L) \times(0,+\infty) \\
u(0, t)=u(L, t)=\varphi(0, t)=\varphi(L, t)=0, & \forall t \geq 0,
\end{aligned}
$$

and proved that the energy associated with (1.3) decays exponentially without imposing the equal wave speed condition. This result is expected in the presence of linear damping terms in both equations. As they mentioned, their aim was to use a method developed by Liu and Zheng [6], which is based on the semigroup theory. Ammar-Khodja et al. [1] considered a linear Timoshenko-type system with 
memory of the form

$$
\begin{aligned}
\rho_{1} \varphi_{t t}-K\left(\varphi_{x}+\psi\right)_{x} & =0, \quad \text { in }(0, L) \times(0,+\infty) \\
\rho_{2} \psi_{t t}-b \psi_{x x}+\int_{0}^{t} g(t-s) \psi_{x x}(s) d s+K\left(\varphi_{x}+\psi\right) & =0, \\
& \text { in }(0, L) \times(0,+\infty)
\end{aligned}
$$

together with homogeneous boundary conditions. They used the multiplier techniques and proved that the system is uniformly stable if and only if the wave speeds are equal $\left(\frac{K}{\rho_{1}}=\frac{b}{\rho_{2}}\right)$ and $g$ decays uniformly. Precisely, they proved an exponential decay if $g$ decays in an exponential rate and polynomially if $g$ decays in a polynomial rate. In [4], Guesmia and Messaoudi investigated the effect of both frictional and viscoelastic dampings. They considered the following system

$$
\begin{aligned}
\varphi_{t t}-\left(\varphi_{x}+\psi\right)_{x} & =0 \\
\psi_{t t}-\psi_{x x}+\varphi_{x}+\psi+\int_{0}^{t} g(t-s)\left(a(x) \psi_{x}(s)\right)_{x} d s+b(x) h\left(\psi_{t}\right) & =0
\end{aligned}
$$

in $(0,1) \times(0,+\infty)$, together with homogeneous boundary conditions. An exponential and polynomial decay result has been established under weaker conditions on the relaxation function $g$ than that in [1]. Santos [13] considered a Timoshenko system and showed that the presence of two feedbacks of memory type at a portion of the boundary stabilizes the system uniformly, and the rate of decay of the energy is of the same order of decay as the relaxation functions. This result has been generalized by Messaoudi and Soufyane [8], where they considered a multidimensional Timoshenko-type system with boundary conditions of memory type and proved energy decay results, for which the usual exponential and polynomial decay rates are only special cases. For more results concerning the controllability of Timoshenko systems, we refer the reader to [2,3,14,17], and [19].

In this paper we are concerned with the following types of Timoshenko systems

$$
\begin{aligned}
& \left\{\begin{array}{l}
a \varphi_{t t}-k\left(\varphi_{x}+\psi\right)_{x}=0, \quad(0,1) \times \mathbb{R}_{+} \\
b \psi_{t t}-d \psi_{x x}+k\left(\varphi_{x}+\psi\right)+h_{2}\left(\psi_{t}\right)=0, \quad(0,1) \times \mathbb{R}_{+} \\
\varphi(0, t)=\psi(0, t)=\psi(1, t)=0, \quad \varphi_{x}(1, t)=-h_{1}\left(\varphi_{t}(1, t)\right), \quad t \geq 0 \\
\varphi(\cdot, 0)=\varphi_{0}, \varphi_{t}(\cdot, 0)=\varphi_{1}, \psi(\cdot, 0)=\psi_{0}, \psi_{t}(\cdot, 0)=\psi_{1} \quad \text { in } \quad(0,1)
\end{array}\right. \\
& \left\{\begin{array}{l}
a \varphi_{t t}-k\left(\varphi_{x}+\psi\right)_{x}+h_{1}\left(\varphi_{t}\right)=0, \quad(0,1) \times \mathbb{R}_{+} \\
b \psi_{t t}-d \psi_{x x}+k\left(\varphi_{x}+\psi\right)+h_{2}\left(\psi_{t}\right)=0, \quad(0,1) \times \mathbb{R}_{+} \\
\varphi(0, t)=\varphi(1, t)=\psi(0, t)=\psi(1, t)=0, \quad t \geq 0 \\
\varphi(\cdot, 0)=\varphi_{0}, \varphi_{t}(\cdot, 0)=\varphi_{1}, \quad \psi(\cdot, 0)=\psi_{0}, \psi_{t}(\cdot, 0)=\psi_{1} \quad \text { in } \quad(0,1)
\end{array}\right.
\end{aligned}
$$


and

$$
\left\{\begin{array}{l}
a \varphi_{t t}-k\left(\varphi_{x}+\psi\right)_{x}=0, \quad(0,1) \times \mathbb{R}_{+} \\
b \psi_{t t}-d \psi_{x x}+k\left(\varphi_{x}+\psi\right)=0, \quad(0,1) \times \mathbb{R}_{+} \\
\varphi(0, t)=0, \quad \psi(1, t)+\varphi_{x}(1, t)=-h_{1}\left(\varphi_{t}(1, t)\right), \quad t \geq 0 \\
\psi(0, t)=0, \quad \psi_{x}(1, t)=-h_{2}\left(\psi_{t}(1, t)\right), \quad t \geq 0 \\
\varphi(\cdot, 0)=\varphi_{0}, \quad \varphi_{t}(\cdot, 0)=\varphi_{1}, \psi(\cdot, 0)=\psi_{0}, \psi_{t}(\cdot, 0)=\psi_{1} \quad \text { in } \quad(0,1)
\end{array}\right.
$$

where $h_{1}$ and $h_{2}$ are specific functions and $a, b, d, k$ are positive constants. These systems describe the transverse vibrations of a beam subjected to a joint effect of two (internal or/and boundary) frictional mechanisms. Our aim is to establish explicit and generalized decay rate results for the energy of these systems, without imposing any restrictive growth assumption near the origin on the damping terms. The results of this paper allow a larger class of functions $h_{1}$ and $h_{2}$, from which the energy decay rates are not necessarily of exponential or polynomial types (see the examples in Section 4).

The proofs of our results are done basically in two steps. In the first step, we use the multiplier method and benefit from [2] and [8] to choose the right multipliers. In the second step, we follow, with necessary modifications dictated by the nature of our systems, the method introduced and used by Martinez [7] to study the wave equations. The paper is organized as follows. In Section 2, we present some notations and material needed for our work. The statements and proofs of the main results are given in Sections 3 and 4. In the last section, we investigate the special case of the polynomial growth.

\section{Preliminaries}

In this section we present some material needed for the proofs of our main results. In the sequel we assume that system (1.6) has a unique solution

$$
\begin{aligned}
& \varphi \in L^{\infty}\left(\mathbb{R}_{+} ; H^{2}(0,1) \cap V\right) \cap W^{1, \infty}\left(\mathbb{R}_{+} ; V\right) \cap W^{2, \infty}\left(\mathbb{R}_{+} ; L^{2}(0,1)\right), \\
& \psi \in L^{\infty}\left(\mathbb{R}_{+} ; H^{2}(0,1) \cap H_{0}^{1}(0,1)\right) \cap W^{1, \infty}\left(\mathbb{R}_{+} ; H_{0}^{1}(0,1)\right) \cap W^{2, \infty}\left(\mathbb{R}_{+} ; L^{2}(0,1)\right),
\end{aligned}
$$

system (1.7) has a unique solution

$\varphi, \psi \in L^{\infty}\left(\mathbb{R}_{+} ; H^{2}(0,1) \cap H_{0}^{1}(0,1)\right) \cap W^{1, \infty}\left(\mathbb{R}_{+} ; H_{0}^{1}(0,1)\right) \cap W^{2, \infty}\left(\mathbb{R}_{+} ; L^{2}(0,1)\right)$, and system (1.8) has a unique solution

$$
\varphi, \psi \in L^{\infty}\left(\mathbb{R}_{+} ; H^{2}(0,1) \cap V\right) \cap W^{1, \infty}\left(\mathbb{R}_{+} ; V\right) \cap W^{2, \infty}\left(\mathbb{R}_{+} ; L^{2}(0,1)\right),
$$

where $V=\left\{v \in H^{1}(0,1): v(0)=0\right\}$. These results can be proved, for initial data in suitable function spaces, using standard arguments such as the Galerkin method.

The following lemma will be of essential use in establishing our main results. 
Lemma 2.1 ([7]). Let $E: \mathbb{R}_{+} \rightarrow \mathbb{R}_{+}$be a nonincreasing function and $\sigma: \mathbb{R}_{+} \rightarrow \mathbb{R}_{+}$ be a strictly increasing $C^{1}$-function, with $\sigma(t) \rightarrow+\infty$ as $t \rightarrow+\infty$.

Assume that there exist $p, q \geq 0$ and $c>0$ such that

$$
\int_{S}^{\infty} \sigma^{\prime}(t) E(t)^{1+p} d t \leq c E(S)^{1+p}+\frac{c E(S)}{\sigma^{q}} \quad 1 \leq S<+\infty .
$$

Then there exist positive constants $k$ and $\omega$ such that

$$
\begin{aligned}
& E(t) \leq k e^{-\omega \sigma(t)} \quad \forall t \geq 1, \quad \text { if } \quad p=q=0 \\
& E(t) \leq \frac{k}{\sigma(t)^{\frac{1+q}{p}}} \quad \forall t \geq 1, \quad \text { if } \quad p>0 .
\end{aligned}
$$

Now, we introduce the energy functional

$$
E(t):=\frac{1}{2} \int_{0}^{1}\left(a \varphi_{t}^{2}+b \psi_{t}^{2}+d \psi_{x}^{2}+k\left(\varphi_{x}+\psi\right)^{2}\right) d x .
$$

We will use $c$, throughout this paper, to denote a generic positive constant which may depend on the initial energy of the solution (see (3.12) for instance).

\section{Decay of energy of system (1.6)}

In this section we state and prove our main result for system (1.6). We consider the following hypothesis on $h_{1}$ and $h_{2}$

$(\mathrm{H} 1) h_{i}: \mathbb{R} \rightarrow \mathbb{R}$ (for $i=1,2$ ) are nondecreasing $C^{1}$ functions such that

$$
\begin{aligned}
H_{i}(|s|) & \leq\left|h_{i}(s)\right| \leq H_{i}^{-1}(|s|) \quad \text { for all } \quad|s| \leq m, \quad i=1,2 \\
c_{1}|s| & \leq\left|h_{1}(s)\right| \leq c_{2}|s| \quad \text { for all }|s| \geq m \\
c_{1}|s| & \leq\left|h_{2}(s)\right| \leq c_{2}|s|^{q} \quad \text { for all }|s| \geq m,
\end{aligned}
$$

where $H_{1}$ and $H_{2}$ are strictly increasing $C^{1}$ functions on $[0,+\infty), H_{1}(0)=$ $H_{2}(0)=0$, the constants $m, c_{1}, c_{2}$ are positive, and $q \geq 1$.

Remark 3.1. Hypothesis (H1) implies that $s h_{i}(s)>0$, for all $s \neq 0, i=1,2$.

Lemma 3.1. Let $(\varphi, \psi)$ be the solution of (1.6). Then the energy functional satisfies

$$
E^{\prime}(t)=-k \varphi_{t}(1, t) h_{1}\left(\varphi_{t}(1, t)\right) d x-\int_{0}^{1} \psi_{t} h_{2}\left(\psi_{t}\right) d x \leq 0
$$

Proof. By multiplying the first two equations in (1.6) by $\varphi_{t}$ and $\psi_{t}$ respectively, integrating over $(0,1)$, and doing some manipulations, we obtain (3.1).

In the next lemma, we use the multiplier $w$, defined by

$$
w(x, t)=-\int_{0}^{x} \psi(s, t) d s, \quad x \in[0,1] .
$$


Lemma 3.2. Let $(\varphi, \psi)$ be the solution of (1.6) and $\sigma: \mathbb{R}_{+} \rightarrow \mathbb{R}_{+}$be a concave nondecreasing $C^{2}$-function. Then, for $T \geq S \geq 0$, the energy functional satisfies

$$
\begin{aligned}
\int_{S}^{T} \sigma^{\prime}(t) E(t)^{2} d t \leq & c E(S)^{2}+c \int_{S}^{T} \sigma^{\prime} E\left(\int_{0}^{1}\left[\psi_{t}^{2}-c^{\prime} \psi h_{2}\left(\psi_{t}\right)\right] d x\right) d t \\
& +c \int_{S}^{T} \sigma^{\prime} E\left(\varphi_{t}^{2}(1, t)+h_{1}^{2}\left(\varphi_{t}(1, t)\right)\right) d t
\end{aligned}
$$

Proof. We multiply the first equation in (1.6) by $\left(x \varphi_{x}+N w\right) \sigma^{\prime} E$ and the second equation by $N \psi \sigma^{\prime} E$, where $N>0$ to be chosen later, integrate over $(0,1) \times(S, T)$, and use integration by parts to get

$$
\begin{aligned}
\int_{S}^{T} \sigma^{\prime}(t) E(t)^{2} d t=-\left[\sigma^{\prime}(t) E(t) \int_{0}^{1}\left(a x \varphi_{x} \varphi_{t}+N a w \varphi_{t}+N b \psi \psi_{t}\right) d x\right]_{S}^{T} \\
\quad+\int_{S}^{T}\left(\sigma^{\prime \prime} E+\sigma^{\prime} E^{\prime}\right)\left(\int_{0}^{1}\left[a x \varphi_{x} \varphi_{t}+N a w \varphi_{t}+N b \psi \psi_{t}\right] d x\right) d t \\
+N a \int_{S}^{T} \sigma^{\prime} E\left(\int_{0}^{1} w_{t} \varphi_{t} d x\right) d t+k \int_{S}^{T} \sigma^{\prime} E\left(\int_{0}^{1}\left(\psi+x \psi_{x}\right)\left(\psi+\varphi_{x}\right) d x\right) d t \\
\quad+N k \int_{S}^{T} \sigma^{\prime} E\left(w(1, t) \varphi_{x}(1, t)\right) d t-\int_{S}^{T} \sigma^{\prime} E\left(\int_{0}^{1}\left(N d-\frac{d}{2}\right) \psi_{x}^{2} d x\right) d t \quad(3.4) \\
+\int_{S}^{T} \sigma^{\prime} E\left(\int_{0}^{1}\left[\left(N b+\frac{b}{2}\right) \psi_{t}^{2}-N \psi h_{2}\left(\psi_{t}\right)\right] d x\right) d t \\
+\int_{S}^{T} \sigma^{\prime} E\left[\frac{a}{2} \varphi_{t}^{2}(1, t)+\frac{k}{2} h_{1}^{2}\left(\varphi_{t}(1, t)\right)\right] d t
\end{aligned}
$$

We exploit Young's, Poincaré's, and Hölder's inequalities, and the fact that

$$
\varphi_{x}^{2} \leq 2\left(\psi+\varphi_{x}\right)^{2}+2 \psi^{2},
$$

to estimate the terms in the right hand side of (3.4) as follows

$$
\text { - } I_{1}:=-\left[\sigma^{\prime}(t) E(t) \int_{0}^{1}\left(a x \varphi_{x} \varphi_{t}+N a w \varphi_{t}+N b \psi \psi_{t}\right) d x\right]_{S}^{T} \text {. }
$$

Since

$$
\begin{aligned}
\int_{0}^{1}\left(a x \varphi_{x} \varphi_{t}+N a w \varphi_{t}+N b \psi \psi_{t}\right) d x & \leq c \int_{0}^{1}\left(\varphi_{x}^{2}+\varphi_{t}^{2}+w^{2}+\psi^{2}+\psi_{t}^{2}\right) d x \\
& \leq c \int_{0}^{1}\left(\left(\psi+\varphi_{x}\right)^{2}+\varphi_{t}^{2}+\psi_{x}^{2}+\psi_{t}^{2}\right) d x \\
& \leq c E(t),
\end{aligned}
$$

then, by the properties of $E$ and $\sigma$, we conclude that

$$
I_{1} \leq c\left|\left[\sigma^{\prime}(t) E(t)^{2}\right]_{S}^{T}\right| \leq c \sigma^{\prime}(S) E(S)^{2} .
$$

- $I_{2}:=\int_{S}^{T}\left(\sigma^{\prime \prime} E+\sigma^{\prime} E^{\prime}\right)\left(\int_{0}^{1}\left[a x \varphi_{x} \varphi_{t}+N a w \varphi_{t}+N b \psi \psi_{t}\right] d x\right) d t$. 
As in above, we conclude that

$$
\begin{aligned}
I_{2} & \leq c\left|\int_{S}^{T} \sigma^{\prime \prime} E^{2} d t\right|+c\left|\int_{S}^{T} \sigma^{\prime} E^{\prime} E d t\right| \\
& \leq c E(S)^{2}\left|\int_{S}^{T} \sigma^{\prime \prime} d t\right|+c \sigma^{\prime}(S)\left|\int_{S}^{T} E E^{\prime} d t\right| \\
& \leq c \sigma^{\prime}(S) E(S)^{2} .
\end{aligned}
$$

- $I_{3}:=N a \int_{S}^{T} \sigma^{\prime} E\left(\int_{0}^{1} w_{t} \varphi_{t} d x\right) d t$

$$
I_{3} \leq \varepsilon \int_{S}^{T} \sigma^{\prime} E\left(\int_{0}^{1} \varphi_{t}^{2} d x\right) d t+C_{\varepsilon} \int_{S}^{T} \sigma^{\prime} E\left(\int_{0}^{1} \psi_{t}^{2} d x\right) d t .
$$

- $I_{4}:=k \int_{S}^{T} \sigma^{\prime} E\left(\int_{0}^{1}\left(\psi+x \psi_{x}\right)\left(\psi+\varphi_{x}\right) d x\right) d t$

$$
I_{4} \leq \varepsilon \int_{S}^{T} \sigma^{\prime} E\left(\int_{0}^{1}\left(\psi+\varphi_{x}\right)^{2} d x\right) d t+C_{\varepsilon} \int_{S}^{T} \sigma^{\prime} E\left(\int_{0}^{1} \psi_{x}^{2} d x\right) d t .
$$

- $I_{5}:=N k \int_{S}^{T} \sigma^{\prime} E w(1, t) \varphi_{x}(1, t) d t$

$$
w^{2}(1, t)=\left(\int_{0}^{1} w_{x} d x\right)^{2} \leq \int_{0}^{1} w_{x}^{2} d x \leq c \int_{0}^{1} \psi_{x}^{2} d x .
$$

Therefore, using the boundary condition in (1.6), we have

$$
I_{5} \leq c \int_{S}^{T} \sigma^{\prime} E\left(\int_{0}^{1} \psi_{x}^{2} d x\right) d t+c N^{2} \int_{S}^{T} \sigma^{\prime} E h_{1}^{2}\left(\varphi_{t}(1, t)\right) d t .
$$

By using our estimates for $I_{1}-I_{5}$ into (3.4) and taking $\varepsilon$ small enough and $N$ large enough, we obtain (3.3).

We are now ready to state and prove the main result for system (1.6).

Theorem 3.3. Assume that (H1) holds. Then there exists a constant $c>0$ such that, for t large, the solution of (1.6) satisfies

$$
E(t) \leq c\left(K^{-1}\left(\frac{1}{t}\right)\right)^{2}
$$

where

$$
K(s)=s\left(H_{1}^{-1}+H_{2}^{-1}\right)^{-1}(s) .
$$

Moreover, if $H_{1}, H_{2}$ are strictly convex on $(0, r)$, for some $r>0$, and $H_{1}^{\prime}(0)=$ $H_{2}^{\prime}(0)=0$, then we have the improved estimate

$$
E(t) \leq c\left(\left(H_{1}^{-1}+H_{2}^{-1}\right)\left(\frac{1}{t}\right)\right)^{2} .
$$


Proof. Let $H_{0}:=\left(H_{1}^{-1}+H_{2}^{-1}\right)^{-1}$ and

$$
\phi(t):=1+\int_{1}^{t} \frac{1}{H_{0}(1 / s)} d s \quad t \geq t^{\prime}
$$

for some $t^{\prime}>\max \left\{1, \frac{1}{m}\right\}$. Then

$$
\phi^{\prime}(t)=\frac{1}{H_{0}(1 / t)}>0 \quad \forall t \geq t^{\prime}, \quad \phi^{\prime}(t) \rightarrow+\infty \quad \text { as } \quad t \rightarrow+\infty,
$$

and $\phi^{\prime}(t)$ is strictly increasing.

Thus, $\phi$ is a convex and strictly increasing $C^{2}$-function, with $\phi(t) \rightarrow+\infty$ as $t \rightarrow+\infty$.

If we set

$$
\sigma_{0}:=\phi^{-1}, \quad t \geq t^{\prime}
$$

then it is easy to check that $\sigma_{0}$ is strictly increasing and $\sigma_{0}^{\prime}(t)=H_{0}\left(1 / \sigma_{0}(t)\right)$ is strictly decreasing. So $\sigma_{0}$ is a concave $C^{2}$-function, with $\sigma_{0}(t) \rightarrow+\infty$ as $t \rightarrow+\infty$.

We use this particular function $\sigma_{0}$, and take $t_{1} \geq t^{\prime}$ such that $\sigma_{0}^{\prime}\left(t_{1}\right)<m$, to estimate the last integrals in (3.3), for $T \geq S \geq t_{1}$, as follows.

1) Estimate for $\int_{S}^{T} \sigma_{0}^{\prime} E\left(\int_{0}^{1} \psi_{t}^{2} d x\right) d t$

We consider the following partition of $(0,1)$

$$
\begin{aligned}
& \Omega_{1}=\left\{x \in(0,1):\left|\psi_{t}\right|>m\right\} \\
& \Omega_{2}=\left\{x \in(0,1):\left|\psi_{t}\right| \leq m \text { and }\left|\psi_{t}\right| \leq H_{2}^{-1}\left(\sigma_{0}^{\prime}(t)\right)\right\} \\
& \Omega_{3}=\left\{x \in(0,1):\left|\psi_{t}\right| \leq m \text { and }\left|\psi_{t}\right|>H_{2}^{-1}\left(\sigma_{0}^{\prime}(t)\right)\right\} .
\end{aligned}
$$

Consequently, we have

$$
\begin{aligned}
& \sigma_{0}^{\prime}(t) \int_{\Omega_{1}} \psi_{t}^{2} d x \leq \frac{1}{c_{1}} \sigma_{0}^{\prime}(t) \int_{0}^{1} \psi_{t} h_{2}\left(\psi_{t}\right) d x \leq-c E^{\prime}(t) \\
& \sigma_{0}^{\prime}(t) \int_{\Omega_{2}} \psi_{t}^{2} d x \leq \sigma_{0}^{\prime}(t)\left(H_{2}^{-1}\left(\sigma_{0}^{\prime}(t)\right)\right)^{2} \leq \sigma_{0}^{\prime}(t)\left(H_{0}^{-1}\left(\sigma_{0}^{\prime}(t)\right)\right)^{2} \\
& \sigma_{0}^{\prime}(t) \int_{\Omega_{3}} \psi_{t}^{2} d x \leq m \int_{\Omega_{3}} H_{2}\left(\left|\psi_{t}\right|\right)\left|\psi_{t}\right| d x \leq m \int_{0}^{1} \psi_{t} h_{2}\left(\psi_{t}\right) d x \leq-m E^{\prime}(t),
\end{aligned}
$$

which gives

$$
\int_{S}^{T} \sigma_{0}^{\prime} E\left(\int_{0}^{1} \psi_{t}^{2} d x\right) d t \leq c E(S)^{2}+c E(S) \int_{S}^{T} \sigma_{0}^{\prime}(t)\left(H_{0}^{-1}\left(\sigma_{0}^{\prime}(t)\right)\right)^{2} d t .
$$


2) Estimate for $\int_{S}^{T} \sigma_{0}^{\prime} E\left(\int_{0}^{1}\left(-c^{\prime} \psi h_{2}\left(\psi_{t}\right)\right) d x\right) d t$

We consider the following partition of $(0,1)$

$$
\begin{aligned}
& \Omega^{1}=\left\{x \in(0,1):\left|\psi_{t}\right|>m\right\} \\
& \Omega^{2}=\left\{x \in(0,1):\left|\psi_{t}\right| \leq m \text { and }\left|\psi_{t}\right| \leq \sigma_{0}^{\prime}(t)\right\} \\
& \Omega^{3}=\left\{x \in(0,1):\left|\psi_{t}\right| \leq m \text { and }\left|\psi_{t}\right|>\sigma_{0}^{\prime}(t)\right\} .
\end{aligned}
$$

Then, using Hölder's, Young's and Poincaré's inequalities, (H1) and the embedding $H_{0}^{1}(0,1) \hookrightarrow L^{r}(0,1)$ for $r \geq 1$, we have

$$
\begin{aligned}
\sigma_{0}^{\prime}(t) \int_{\Omega^{1}} \psi h_{2}\left(\psi_{t}\right) d x & \leq \sigma_{0}^{\prime}(t)\left(\int_{\Omega^{1}}|\psi|^{q+1} d x\right)^{\frac{1}{q+1}}\left(\int_{\Omega^{1}}\left|h_{2}\left(\psi_{t}\right)\right|^{1+\frac{1}{q}} d x\right)^{\frac{q}{q+1}} \\
& \leq c \sigma_{0}^{\prime}(t)\left(\int_{0}^{1} \psi_{x}^{2} d x\right)^{\frac{1}{2}}\left(\int_{\Omega^{1}} \psi_{t} h_{2}\left(\psi_{t}\right) d x\right)^{\frac{q}{q+1}} \\
& \leq c \sigma_{0}^{\prime}(t) E(t)^{\frac{1}{2}}\left(-E^{\prime}(t)\right)^{\frac{q}{q+1}} \leq c \sigma_{0}^{\prime}(t)\left[\varepsilon E(t)^{\frac{q+1}{2}}-C_{\varepsilon} E^{\prime}(t)\right] \\
& \leq c \varepsilon \sigma_{0}^{\prime}(t) E(t)-C_{\varepsilon} E^{\prime}(t) . \\
\sigma_{0}^{\prime}(t) \int_{\Omega^{2}} \psi h_{2}\left(\psi_{t}\right) d x & \leq \varepsilon \sigma_{0}^{\prime}(t) \int_{\Omega^{2}} \psi^{2} d x+C_{\varepsilon} \sigma_{0}^{\prime}(t) \int_{\Omega^{2}} h_{2}\left(\psi_{t}\right)^{2} d x \\
& \leq c \varepsilon \sigma_{0}^{\prime}(t) E(t)+C_{\varepsilon} \sigma_{0}^{\prime}(t)\left(H_{2}^{-1}\left(\sigma_{0}^{\prime}(t)\right)\right)^{2} \\
& \leq c \varepsilon \sigma_{0}^{\prime}(t) E(t)+C_{\varepsilon} \sigma_{0}^{\prime}(t)\left(H_{0}^{-1}\left(\sigma_{0}^{\prime}(t)\right)\right)^{2} . \\
\sigma_{0}^{\prime}(t) \int_{\Omega^{3}} \psi h_{2}\left(\psi_{t}\right) d x & \leq \varepsilon \sigma_{0}^{\prime}(t) \int_{\Omega^{3}} \psi^{2} d x+C_{\varepsilon} \sigma_{0}^{\prime}(t) \int_{\Omega^{3}} h_{2}\left(\psi_{t}\right)^{2} d x \\
& \leq c \varepsilon \sigma_{0}^{\prime}(t) E(t)+C_{\varepsilon} H_{2}^{-1}(m) \int_{0}^{1} \psi_{t} h_{2}\left(\psi_{t}\right) d x \\
& \leq c \varepsilon \sigma_{0}^{\prime}(t) E(t)-C_{\varepsilon} E^{\prime}(t) .
\end{aligned}
$$

A combination of all the above leads to

$$
\begin{aligned}
& \int_{S}^{T} \sigma_{0}^{\prime} E\left(\int_{0}^{1}\left(-c^{\prime} \psi h_{2}\left(\psi_{t}\right)\right) d x\right) d t \\
& \quad \leq c \varepsilon \int_{S}^{T} \sigma_{0}^{\prime} E^{2} d t+C_{\varepsilon} E(S)^{2}+C_{\varepsilon} E(S) \int_{S}^{T} \sigma_{0}^{\prime}(t)\left(H_{0}^{-1}\left(\sigma_{0}^{\prime}(t)\right)\right)^{2} d t .
\end{aligned}
$$

3) Estimate for $\int_{S}^{T} \sigma_{0}^{\prime} E \varphi_{t}^{2}(1, t) d t$

By considering the following cases

$$
\begin{aligned}
& C 1:\left|\varphi_{t}(1, t)\right|>m \\
& C 2:\left|\varphi_{t}(1, t)\right| \leq m \quad \text { and } \quad\left|\varphi_{t}(1, t)\right| \leq H_{1}^{-1}\left(\sigma_{0}^{\prime}(t)\right) \text {, } \\
& C 3:\left|\varphi_{t}(1, t)\right| \leq m \quad \text { and } \quad\left|\varphi_{t}(1, t)\right|>H_{1}^{-1}\left(\sigma_{0}^{\prime}(t)\right) \text {, }
\end{aligned}
$$


we deduce, as in the above, that

$$
\int_{S}^{T} \sigma_{0}^{\prime} E \varphi_{t}^{2}(1, t) d t \leq c E(S)^{2}+c E(S) \int_{S}^{T} \sigma_{0}^{\prime}(t)\left(H_{0}^{-1}\left(\sigma_{0}^{\prime}(t)\right)\right)^{2} d t .
$$

4) Estimate for $\int_{S}^{T} \sigma_{0}^{\prime} E h_{1}^{2}\left(\varphi_{t}(1, t)\right) d t$

We consider the following cases

$$
\begin{aligned}
& C^{\prime} 1:\left|\varphi_{t}(1, t)\right|>m \\
& C^{\prime} 2:\left|\varphi_{t}(1, t)\right| \leq m \quad \text { and } \quad\left|\varphi_{t}(1, t)\right| \leq \sigma_{0}^{\prime}(t) \text {, } \\
& C^{\prime} 3:\left|\varphi_{t}(1, t)\right| \leq m \quad \text { and } \quad\left|\varphi_{t}(1, t)\right|>\sigma_{0}^{\prime}(t) \text {, }
\end{aligned}
$$

and we similarly obtain

$$
\int_{S}^{T} \sigma_{0}^{\prime} E h_{1}^{2}\left(\varphi_{t}(1, t)\right) d t \leq c E(S)^{2}+c E(S) \int_{S}^{T} \sigma_{0}^{\prime}(t)\left(H_{0}^{-1}\left(\sigma_{0}^{\prime}(t)\right)\right)^{2} d t .
$$

Combining (3.3), (3.10), (3.13)-(3.15) and taking $\varepsilon$ small enough lead to

$$
\begin{aligned}
\int_{S}^{\infty} \sigma_{0}^{\prime}(t) E(t)^{2} d t & \leq c E(S)^{2}+c E(S) \int_{S}^{\infty} \sigma_{0}^{\prime}(t)\left(H_{0}^{-1}\left(\sigma_{0}^{\prime}(t)\right)\right)^{2} d t \\
& =c E(S)^{2}+c E(S) \int_{\sigma_{0}(S)}^{\infty}\left(H_{0}^{-1}\left(H_{0}\left(\frac{1}{s}\right)\right)\right)^{2} d s \\
& =c E(S)^{2}+\frac{c E(S)}{\sigma_{0}(S)} .
\end{aligned}
$$

Lemma 2.1, then gives

$$
E(t) \leq \frac{c}{\sigma_{0}(t)^{2}} \quad \forall t \geq t_{1} .
$$

To obtain (3.5), we take $s_{0}>t^{\prime}$ such that $H_{0}\left(\frac{1}{s_{0}}\right) \leq 1$. Since $H_{0}$ is increasing and $K(s)=s H_{0}(s)$, we have

$$
\sigma_{0}^{-1}(s) \leq 1+(s-1) \frac{1}{H_{0}\left(\frac{1}{s}\right)} \leq \frac{s}{H_{0}\left(\frac{1}{s}\right)}=\frac{1}{K\left(\frac{1}{s}\right)} \quad \forall s \geq s_{0} .
$$

So, with $t=\frac{1}{K\left(\frac{1}{s}\right)}$, we easily see that

$$
\frac{1}{\sigma_{0}(t)} \leq K^{-1}\left(\frac{1}{t}\right) \quad \forall t \geq t^{\prime}
$$

Therefore, using (3.16), estimate (3.5) is established.

To prove (3.6), we assume, without loss of generality, that $r=m$. In fact, if $r<m$ and $r \leq|s| \leq m$, then, using (H1), we have, for $i=1,2, q_{1}=1$, and $q_{2}=q$,

$$
\left|h_{i}(s)\right| \leq \frac{H_{i}^{-1}(|s|)}{|s|^{q_{i}}}|s|^{q_{i}} \leq \frac{H_{i}^{-1}(m)}{r^{q_{i}}}|s|^{q_{i}}
$$

and

$$
\left|h_{i}(s)\right| \geq \frac{H_{i}(|s|)}{|s|}|s| \geq \frac{H_{i}(r)}{m}|s| .
$$


This implies that

$$
\begin{aligned}
H_{i}(|s|) & \leq\left|h_{i}(s)\right| \leq H_{i}^{-1}(|s|) \quad \text { for all }|s| \leq r, i=1,2 \\
c_{1}^{\prime}|s| & \leq\left|h_{1}(s)\right| \leq c_{2}^{\prime}|s| \quad \text { for all }|s| \geq r \\
c_{1}^{\prime}|s| & \leq\left|h_{2}(s)\right| \leq c_{2}^{\prime}|s|^{q} \quad \text { for all }|s| \geq r,
\end{aligned}
$$

which justifies our assumption $(r=m)$.

Since $H_{1}(0)=H_{2}(0)=H_{1}^{\prime}(0)=H_{2}^{\prime}(0)=0$ and, for $s>0$,

$$
0<K_{0}(s)=\frac{H_{0}(s)}{s}=\frac{\left(H_{1}^{-1}+H_{2}^{-1}\right)^{-1}(s)}{s} \leq \frac{H_{i}(s)}{s}, \quad i=1,2,
$$

then $H_{0}^{\prime}(0)=K_{0}(0)=0$. Also, one can easily conclude that $H_{0}$ is strictly convex on $(0, m)$. Then, using the Mean value Theorem and the strict convexity of $H_{i}$, $i=0,1,2$, on $(0, m)$, we deduce that

$$
K_{i}(s)=\frac{H_{i}(s)}{s}, \quad i=0,1,2,
$$

are strictly increasing on $(0, m)$.

Now, we take $\sigma_{0}=\phi^{-1}$, where

$$
\phi(t):=1+\int_{1}^{t} \frac{1}{K_{0}(1 / s)} d s \quad t \geq t^{\prime} .
$$

In this case, we replace (3.9) and (3.11) by

$$
\begin{aligned}
& \Omega_{1}=\left\{x \in(0,1):\left|\psi_{t}\right|>m\right\} \\
& \Omega_{2}=\left\{x \in(0,1):\left|\psi_{t}\right| \leq m \text { and }\left|\psi_{t}\right| \leq K_{2}^{-1}\left(\sigma_{0}^{\prime}(t)\right)\right\} \\
& \Omega_{3}=\left\{x \in(0,1):\left|\psi_{t}\right| \leq m \text { and }\left|\psi_{t}\right|>K_{2}^{-1}\left(\sigma_{0}^{\prime}(t)\right)\right\}
\end{aligned}
$$

and

$$
\begin{aligned}
& \Omega^{1}=\left\{x \in(0,1):\left|\psi_{t}\right|>m\right\} \\
& \Omega^{2}=\left\{x \in(0,1):\left|\psi_{t}\right| \leq m \text { and } H_{2}^{-1}\left(\left|\psi_{t}\right|\right) \leq K_{2}^{-1}\left(\sigma_{0}^{\prime}(t)\right)\right\} \\
& \Omega^{3}=\left\{x \in(0,1):\left|\psi_{t}\right| \leq m \text { and } H_{2}^{-1}\left(\left|\psi_{t}\right|\right)>K_{2}^{-1}\left(\sigma_{0}^{\prime}(t)\right)\right\} .
\end{aligned}
$$

Consequently, we arrive at

$$
\begin{aligned}
\sigma_{0}^{\prime}(t) \int_{\Omega_{3}} \psi_{t}^{2} d x & \leq \int_{\Omega_{3}} K_{2}\left(\left|\psi_{t}\right|\right) \psi_{t}^{2} d x \\
& =\int_{\Omega_{3}} H_{2}\left(\left|\psi_{t}\right|\right)\left|\psi_{t}\right| d x \\
& \leq \int_{0}^{1} \psi_{t} h_{2}\left(\psi_{t}\right) d x \leq-E^{\prime}(t)
\end{aligned}
$$




$$
\begin{aligned}
\sigma_{0}^{\prime}(t) \int_{\Omega^{3}} h_{2}\left(\psi_{t}\right)^{2} d x & \leq \int_{\Omega^{3}} K_{2}\left(H_{2}^{-1}\left(\left|\psi_{t}\right|\right)\right) H_{2}^{-1}\left(\left|\psi_{t}\right|\right)\left|h_{2}\left(\psi_{t}\right)\right| d x \\
& =\int_{0}^{1} \psi_{t} h_{2}\left(\psi_{t}\right) d x \leq-E^{\prime}(t) .
\end{aligned}
$$

The other cases can be dealt with similarly. Then, the same reasoning leads to $(3.6)$.

\section{Decay of energy of systems (1.7) and (1.8)}

In this section we state and prove our main results for systems (1.7) and (1.8). To achieve this goal, we consider the following hypothesis on $h_{1}$ and $h_{2}$

(H2) $h_{i}: \mathbb{R} \rightarrow \mathbb{R}$ (for $i=1,2$ ) are nondecreasing $C^{1}$ functions such that

$$
\begin{aligned}
H_{i}(|s|) & \leq\left|h_{i}(s)\right| \leq H_{i}^{-1}(|s|) \quad \text { for all } \quad|s| \leq m, \quad i=1,2 \\
c_{1}|s| & \leq\left|h_{i}(s)\right| \leq c_{2}|s|^{q} \quad \text { for all } \quad|s| \geq m, \quad i=1,2
\end{aligned}
$$

where $H_{1}$ and $H_{2}$ are strictly increasing $C^{1}$ functions on $[0,+\infty), H_{1}(0)=$ $H_{2}(0)=0, m, c_{1}, c_{2}$ are positive constants, $q \geq 1$ for system (1.7) and $q=1$ for (1.8).

Remark 4.1. Hypothesis (H2) implies that $s h_{i}(s)>0$, for all $s \neq 0$.

It is easy to check that the energy functional for system (1.7) satisfies

$$
E^{\prime}(t)=-\int_{0}^{1} \varphi_{t} h_{1}\left(\varphi_{t}\right) d x-\int_{0}^{1} \psi_{t} h_{2}\left(\psi_{t}\right) d x \leq 0
$$

and for system (1.8)

$$
E^{\prime}(t)=-k \varphi_{t}(1, t) h_{1}\left(\varphi_{t}(1, t)\right)-d \psi_{t}(1, t) h_{2}\left(\psi_{t}(1, t)\right) \leq 0 .
$$

Theorem 4.1. Assume that (H2) holds. Then there exists a constant $c>0$ such that, for $t$ large, the solutions of (1.7) and (1.8) satisfy

$$
E(t) \leq c\left(K^{-1}\left(\frac{1}{t}\right)\right)^{2}
$$

where $K(s)=s\left(H_{1}^{-1}+H_{2}^{-1}\right)^{-1}(s)$.

Moreover, if $H_{1}, H_{2}$ are strictly convex on $(0, r)$, for some $r>0$, and $H_{1}^{\prime}(0)=$ $H_{2}^{\prime}(0)=0$, then we have the improved estimate

$$
E(t) \leq c\left(\left(H_{1}^{-1}+H_{2}^{-1}\right)\left(\frac{1}{t}\right)\right)^{2} .
$$

Proof. We define $\sigma_{0}$ as in (3.7) and (3.8), and so $\sigma_{0}$ is a strictly increasing concave $C^{2}$-function, with $\sigma_{0}(t) \rightarrow+\infty$ as $t \rightarrow+\infty$. 


\section{A) System (1.7)}

By multiplying the first two equations in (1.7) by $\sigma_{0}^{\prime} E \varphi$ and $\sigma_{0}^{\prime} E \psi$ respectively, integrating over $(0,1) \times(S, T)$, and using integration by parts, we obtain

$$
\begin{aligned}
2 \int_{S}^{T} \sigma_{0}^{\prime}(t) E(t)^{2} d t= & -\left[\sigma_{0}^{\prime}(t) E(t) \int_{0}^{1}\left(a \varphi \varphi_{t}+b \psi \psi_{t}\right) d x\right]_{S}^{T} \\
& +\int_{S}^{T}\left(\sigma_{0}^{\prime \prime} E+\sigma_{0}^{\prime} E^{\prime}\right)\left(\int_{0}^{1}\left[a \varphi \varphi_{t}+b \psi \psi_{t}\right] d x\right) d t \\
& +\int_{S}^{T} \sigma_{0}^{\prime} E\left(\int_{0}^{1}\left[2 a \varphi_{t}^{2}-\varphi h_{1}\left(\varphi_{t}\right)+2 b \psi_{t}^{2}-\psi h_{2}\left(\psi_{t}\right)\right] d x\right) d t
\end{aligned}
$$

Similar computations as in Lemma 3.2 lead to

$$
\begin{aligned}
\int_{S}^{T} \sigma_{0}^{\prime}(t) E(t)^{2} d t \leq c E(S)^{2} & \\
& +c \int_{S}^{T} \sigma_{0}^{\prime} E\left(\int_{0}^{1}\left[2 a \varphi_{t}^{2}-\varphi h_{1}\left(\varphi_{t}\right)+2 b \psi_{t}^{2}-\psi h_{2}\left(\psi_{t}\right)\right] d x\right) d t .
\end{aligned}
$$

\section{B) System (1.8)}

We multiply the equations in (1.8) by $\left[(N+1) x \varphi_{x}-\frac{N}{2} \varphi\right] \sigma_{0}^{\prime} E$ and $\left[(N+1) x \psi_{x}+\right.$ $\left.\frac{N}{2} \psi\right] \sigma_{0}^{\prime} E$ respectively, where $N>0$ to be suitably chosen, and perform some manipulations to get

$$
\begin{aligned}
\int_{S}^{T} \sigma_{0}^{\prime}(t) & E(t)^{2} d t=\int_{S}^{T} \sigma_{0}^{\prime} E\left(\int_{0}^{1} k \psi\left(\varphi_{x}+\psi\right) d x-N \int_{0}^{1}\left(a \varphi_{t}^{2}+d \psi_{x}^{2}\right) d x\right) d t \\
& -\left[\sigma_{0}^{\prime}(t) E(t) \int_{0}^{1}\left[(N+1)\left(a x \varphi_{x} \varphi_{t}+b x \psi_{x} \psi_{t}\right)+\frac{N}{2}\left(b \psi \psi_{t}-a \varphi \varphi_{t}\right)\right] d x\right]_{S}^{T} \\
& +\int_{S}^{T}\left(\sigma_{0}^{\prime \prime} E+\sigma_{0}^{\prime} E^{\prime}\right)\left(\int _ { 0 } ^ { 1 } \left[(N+1)\left(a x \varphi_{x} \varphi_{t}+b x \psi_{x} \psi_{t}\right)\right.\right. \\
& \left.\left.+\frac{N}{2}\left(b \psi \psi_{t}-a \varphi \varphi_{t}\right)\right] d x\right) d t \\
& +\frac{N}{2} \int_{S}^{T} \sigma_{0}^{\prime} E\left[k \varphi(1, t) h_{1}\left(\varphi_{t}(1, t)\right)-d \psi(1, t) h_{2}\left(\psi_{t}(1, t)\right)\right] d t \\
& +(N+1) k \int_{S}^{T} \sigma_{0}^{\prime} E \psi(1, t) h_{1}\left(\varphi_{t}(1, t)\right) d t \\
& +\frac{N+1}{2} \int_{S}^{T} \sigma_{0}^{\prime} E\left[a \varphi_{t}^{2}(1, t)+k h_{1}^{2}\left(\varphi_{t}(1, t)\right)\right. \\
& \left.+b \psi_{t}^{2}(1, t)+d h_{2}^{2}\left(\psi_{t}(1, t)\right)\right] d t
\end{aligned}
$$


The terms in the right hand side of (4.6) can be estimated similarly as in Lemma 3.2 and we obtain

$$
\begin{aligned}
\int_{S}^{T} \sigma_{0}^{\prime}(t) E(t)^{2} d t \leq & c E(S)^{2} \\
& +c \int_{S}^{T} \sigma_{0}^{\prime} E\left[\varphi_{t}^{2}(1, t)+h_{1}^{2}\left(\varphi_{t}(1, t)\right)+\psi_{t}^{2}(1, t)\right. \\
& \left.+h_{2}^{2}\left(\psi_{t}(1, t)\right)\right] d t
\end{aligned}
$$

By repeating the same procedures as in Theorem 3.3, we estimate the integral term in (4.5) or in (4.7). Consequently, (4.3) and (4.4) are established.

Examples. We give some examples to illustrate the energy decay rates obtained by our results.

(1) Between polynomial and exponential growth

If $H_{1}(s)=H_{2}(s)=e^{-(\ln s)^{2}}$ near zero. Then, we have the following energy decay rate

$$
E(t) \leq c e^{-2(\ln t)^{\frac{1}{2}}}
$$

\section{(2) Exponential growth}

If $H_{1}(s)=H_{2}(s)=e^{-1 / s}$ near zero. Then, we have the following energy decay rate

$$
E(t) \leq \frac{c}{(\ln (t))^{2}}
$$

(3) Faster than exponential growth

If $H_{1}(s)=H_{2}(s)=e^{-e^{1 / s}}$ near zero. Then, we have the following energy decay rate

$$
E(t) \leq \frac{c}{(\ln (\ln (t)))^{2}}
$$

\section{The case of the polynomial growth}

As a special case of (H1) on the system (1.6), we assume that there exist constants $c_{1}, c_{2}>0$ and $q_{1}, q_{2} \geq 1$ such that

$$
c_{1} \min \left\{|s|,|s|^{q_{i}}\right\} \leq\left|h_{i}(s)\right| \leq c_{2} \max \left\{|s|,|s|^{1 / q_{i}}\right\} \quad i=1,2
$$

According to Theorem 3.3, we have the following estimate

$$
E(t) \leq \frac{c}{t^{2 / q}} .
$$

where $q=\max \left\{q_{1}, q_{2}\right\}$. However, we can obtain a better decay rate as follows. 
We multiply the first equation in (1.6) by $\left(x \varphi_{x}+N w\right) E^{\frac{q-1}{2}}$ and the second equation by $N \psi E^{\frac{q-1}{2}}$ for $q=\max \left\{q_{1}, q_{2}\right\}$. Consequently, by similar computations as in Lemma 3.2, for $\sigma(t)=t$, we obtain

$$
\begin{aligned}
\int_{S}^{T} E(t)^{1+\frac{q-1}{2}} d t \leq & c E(S)^{1+\frac{q-1}{2}}+c \int_{S}^{T} E^{\frac{q-1}{2}}\left(\int_{0}^{1}\left[\psi_{t}^{2}-c^{\prime} \psi h_{2}\left(\psi_{t}\right)\right] d x\right) d t \\
& +c \int_{S}^{T} E^{\frac{q-1}{2}}\left(\varphi_{t}^{2}(1, t)+h_{1}^{2}\left(\varphi_{t}(1, t)\right)\right) d t \\
\leq & c E(S)^{1+\frac{q-1}{2}}+\varepsilon \int_{S}^{T} E^{1+\frac{q-1}{2}} d t \\
& +C_{\varepsilon} \int_{S}^{T} E^{\frac{q-1}{2}}\left(\int_{0}^{1}\left[\psi_{t}^{2}+h_{2}\left(\psi_{t}\right)^{2}\right] d x\right) d t \\
& +c \int_{S}^{T} E^{\frac{q-1}{2}}\left(\varphi_{t}^{2}(1, t)+h_{1}^{2}\left(\varphi_{t}(1, t)\right)\right) d t .
\end{aligned}
$$

By choosing $\varepsilon$ small enough and using (5.1), we infer

$$
\begin{aligned}
\int_{S}^{T} E(t)^{1+\frac{q-1}{2}} d t \leq & c E(S)^{1+\frac{q-1}{2}} \\
& +c \int_{S}^{T} E^{\frac{q-1}{2}}\left(\int_{0}^{1}\left[\left(\psi_{t} h_{2}\left(\psi_{t}\right)\right)^{\frac{2}{q_{2}+1}}+\psi_{t} h_{2}\left(\psi_{t}\right)\right] d x\right) d t \\
& +c \int_{S}^{T} E^{\frac{q-1}{2}}\left(\left(\varphi_{t}(1, t) h_{1}\left(\varphi_{t}(1, t)\right)\right)^{\frac{2}{q_{1}+1}}\right. \\
& \left.+\varphi_{t}(1, t) h_{1}\left(\varphi_{t}(1, t)\right)\right) d t
\end{aligned}
$$

Case 1: $\left(q_{1}, q_{2}\right)=(1,1)$

In this case, we clearly have

$$
\int_{S}^{T} E(t) d t \leq c E(S)+c \int_{S}^{T}\left(-E^{\prime}(t)\right) d t \leq c E(S) .
$$

Case 2: $\left(q_{1}, q_{2}\right) \neq(1,1)$

The use of Hölder's and Young's inequalities, in (5.2), yields

$$
\begin{aligned}
\int_{S}^{T} E(t)^{1+\frac{q-1}{2}} d t & \leq c E(S)^{1+\frac{q-1}{2}}+c \int_{S}^{T} E^{\frac{q-1}{2}}\left[\left(-E^{\prime}\right)^{\frac{2}{q_{1}+1}}+\left(-E^{\prime}\right)^{\frac{2}{q_{2}+1}}\right] d t \\
& \leq c E(S)^{1+\frac{q-1}{2}}+C_{\delta} E(S)+\delta \int_{S}^{T} E^{1+\frac{q-1}{2}} d t .
\end{aligned}
$$

Hence, choosing $\delta$ small enough, we find

$$
\int_{S}^{T} E(t)^{1+\frac{q-1}{2}} d t \leq c E(S)^{1+\frac{q-1}{2}}+c E(S) .
$$


Consequently, (5.3), (5.4), and Lemma 2.1 lead to

$$
\begin{aligned}
& E(t) \leq c e^{-w t}, \quad \text { if } \quad\left(q_{1}, q_{2}\right)=(1,1) \\
& E(t) \leq \frac{c}{t^{\frac{2}{q-1}}}, \quad q=\max \left\{q_{1}, q_{2}\right\}, \quad \text { if } \quad\left(q_{1}, q_{2}\right) \neq(1,1) .
\end{aligned}
$$

Remark 5.1. By the same way, under the condition (5.1), we obtain (5.5)-(5.6) for the systems (1.7) and (1.8).

Remark 5.2. We note that our results allow a larger class of functions $h_{1}, h_{2}$. In fact, the usual exponential and polynomial decay estimates are only special cases. These results improve and generalize those established by Kim and Renardy [5], Yan [18], and Raposo et al. [12], and extend the decay results established for the wave equations by Martinez [7] to the Timoshenko systems.

\section{Acknowledgements}

The authors thank the referees for the valuable suggestions which improved a lot this work. This work has been funded by KFUPM under Project \#FT080003.

\section{References}

[1] F. Ammar-Khodja, A. Benabdallah, J. E. Muñoz Rivera, and R. Racke, Energy decay for Timoshenko systems of memory type, J. Differential Equations 194 no. 1 (2003), $82-115$.

[2] F. Ammar-Khodja, S. Kerbal, and A.E. Soufyane, Stabilization of the nonuniform Timoshenko beam, J. Math. Anal. Appl. 327 no. 1 (2007), 525-538.

[3] D.-X. Feng, D.-H. Shi, and W. Zhang, Boundary feedback stabilization of Timoshenko beam with boundary dissipation, Sci. China Ser. A 41 no. 5 (1998), 483-490.

[4] A. Guesmia and S. A. Messaoudi, Frictional versus viscoelastic damping for Timoshenko-type systems, Proceedings of the 5th UAE Math-Day Conference, MHBM Sharif (ed) (2007) Aardvark Global, Isbn 978-1-4276-2016-3.

[5] J. U. Kim and Y. Renardy, Boundary control of the Timoshenko beam, SIAM J. Control Optim. 25 no. 6 (1987),1417-1429.

[6] Z. Liu and S. Zheng, Semigroups associated with dissipative systems, Chapman \& Hall/CRC, 1999

[7] P. Martinez, A new method to obtain decay rate estimates for dissipative systems with localized damping, Rev. Mat. Complut. 12 no. 1 (1999), 251-283.

[8] S. A. Messaoudi and A. Soufyane, Boundary stabilization of a nonlinear system of Timoshenko type, Nonlinear Analysis 67 (2007), 2107-212.

[9] J. E. Muñoz Rivera and R. Racke, Mildly dissipative nonlinear Timoshenko systemsglobal existence and exponential stability, J. Math. Anal. Appl. 276 (2002), 248-276.

[10] J. E. Muñoz Rivera and R. Racke, Global stability for damped Timoshenko systems, Discrete Contin. Dyn. Syst. 9 no. 6 (2003), 1625-1639. 
[11] J.E. Muñoz Rivera and R. Racke, Timoshenko systems with indefinite damping, J. Math Anal. Appl. 341 (2008), 1068-1083.

[12] C. A. Raposo, J. Ferreira, M. L. Santos, and N. N. O. Castro, Exponential stability for the Timoshenko system with two weak dampings, Applied Math Letters 18 (2005), $535-541$

[13] M. Santos, Decay rates for solutions of a Timoshenko system with a memory condition at the boundary, Abstr. Appl. Anal. 7 no. 10 (2002), 531-546.

[14] D.-H. Shi and D.-X. Feng, Exponential decay rate of the energy of a Timoshenko beam with locally distributed feedback, ANZIAM J. 44 no. 2 (2002), 205-220.

[15] A. Soufyane and A. Wehbe, Uniform stabilization for the Timoshenko beam by a locally distributed damping, Elec. J. Differential Equations no. 29 (2003), 1-14.

[16] S. Timoshenko, On the correction for shear of the differential equation for transverse vibrations of prismatic bars, Philosophical magazine 41 (1921), 744-746.

[17] G.-Q. Xu and S.-P. Yung, Stabilization of Timoshenko beam by means of pointwise controls, ESAIM, Control Optim. Calc. Var. 9 (2003), 579-600.

[18] Q.-X. Yan, Boundary stabilization of Timoshenko beam, Systems Science and Mathematical Sciences 13 no. 4 (2000), 376-384.

[19] Q-X Yan, H-C Zou, and D-X Feng, Nonlinear boundary stabilization of nonuniform Timoshenko beam, Acta Mathematicae Applicatae Sinica 19 no. 2 (2003), 239-246.

Salim A. Messaoudi

Department of Mathematics and Statistics

KFUPM

Dhahran 31261

Saudi Arabia

e-mail: messaoud@kfupm.edu.sa

Muhammad I. Mustafa

King Fahd University of Petroleum and Minerals

Department of Mathematics and Statistics

Dhahran 31261

Saudi Arabia

e-mail: mohmim@kfupm.edu .

Received: 8 December 2007.

Revised: 25 March 2008.

Accepted: 20 May 2008. 